\title{
Dynamic modeling of heat pumps for ancillary services in local district heating concepts
}

\author{
Mads Pagh Nielsen Kim Sørensen \\ Department of Energy Technology, Aalborg University, Denmark, \{mpn, kso\} @et . aau . dk
}

\begin{abstract}
This paper aims to describe the steady-state and dynamic heat pump models developed to study their abilities in ancillary services as well as the interconnection between these, electrical boilers and thermal storage with the aim to balance power and heat production for a given case.

An hourly steady-state system model was developed to understand the overall operational characteristics of the system for a given heat demand case in the area KoltHasselager-Ormslev near Aarhus in Denmark. The model showed an annual average COP above 3,5 for a serial connected heat pump system.

Detailed thermal and dynamical models of the heat pump system were developed. The models show that it will be possible to use heat pumps successfully in ancillary services. The turn-up is unproblematic but the turn down of the heat pump will be limited in a nonliquid overfed system due to risk of liquid formation in the evaporator, requiring additional heating.
\end{abstract}

Keywords: heat pumps, dynamic modeling, district heating, thermal properties, ancillary services.

\section{Introduction}

The Danish heating sector has successfully evolved over the past 50 years. Today, more than $60 \%$ of the domestic heating in Denmark is supplied by district heating. Numerous developments like urbanization, city densification, reduction in heating consumption through better insulation standards etc. challenges the efficient operation of the district heating systems.

Simultaneously, the development of wind farms has created a surplus of electricity in Denmark requiring power balancing. Heat pumps coupled with electrical boilers and heat storage could provide such balancing maintaining an efficient heat supply system. For automatic frequency response reserve actions the response time and operation of the heat pump should meet certain standards (max. $150 \mathrm{~s}$ up-/down regulation for Frequency Containment Services) depending on the way the heat pump acts as backup for power and net frequency fluctuations. The downward and upward regulation compensation could potentially greatly benefit the economy of heat pump systems if the dynamic response is fast (Energidataservice, 2020).

The "Local Heating Concepts" ForskEL project funded by the Danish Government has through detailed inputs from several asset owners investigated the possibilities of integrating localized heat pumps in district heating and power grids. Through laboratory emulations at Aalborg University, Department of Energy Technology, the models will later be coupled with real power system components enabling a realistic dynamic emulation.

There are not many works published on the operation of large-scale heat pumps for ancillary services in Denmark. However, recently, (Meesenburg et al., 2020) published a paper on the optimization of heat pumps for this purpose.

Detailed heat pump models designed for both parallel and serial coupling have been developed and simulations have been carried out. As a case, the district heating grid at Kolt-Hasselager-Ormslev near Aarhus has been chosen. The system operation and some system configurations have been investigated.

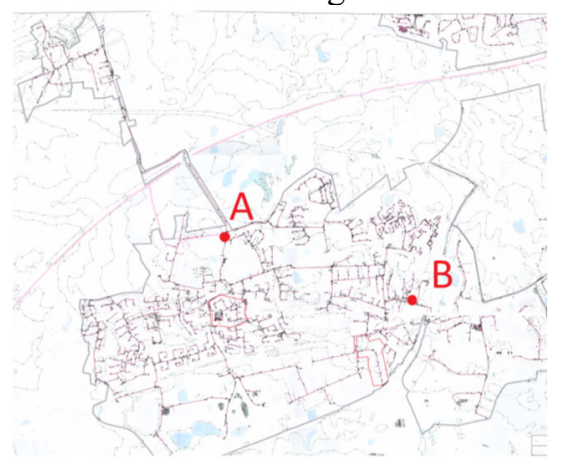

Figure 1. Kolt-Hasselager Ormslev district heating grid.

Currently, the district heating area is supplied by a largescale plate heat exchanger linked to the central CHPplant in Aarhus and industrial heat suppliers (A) and three oil-fired backup boilers (B). In the coming years the district heating demand will increase significantly requiring additional capacity. The supplier would also like to replace the oil-fired boilers with heat pumps.

\section{Traditional operational modeling}

Degree day corrected heat demand data for 2017 in the area is shown in Figure 2 along with the heat rate demand curve. The heat demand has furthermore been corrected to reflect the expected future heat demand as predicted by the heat supplier AVA (AffaldVarme Aarhus). The area around Ormslev shown in the upper left corner of Figure 1 is expected to be expanded significantly with several new areas with family dwellings thus requiring a total of around 5-10 MW additional heat. 

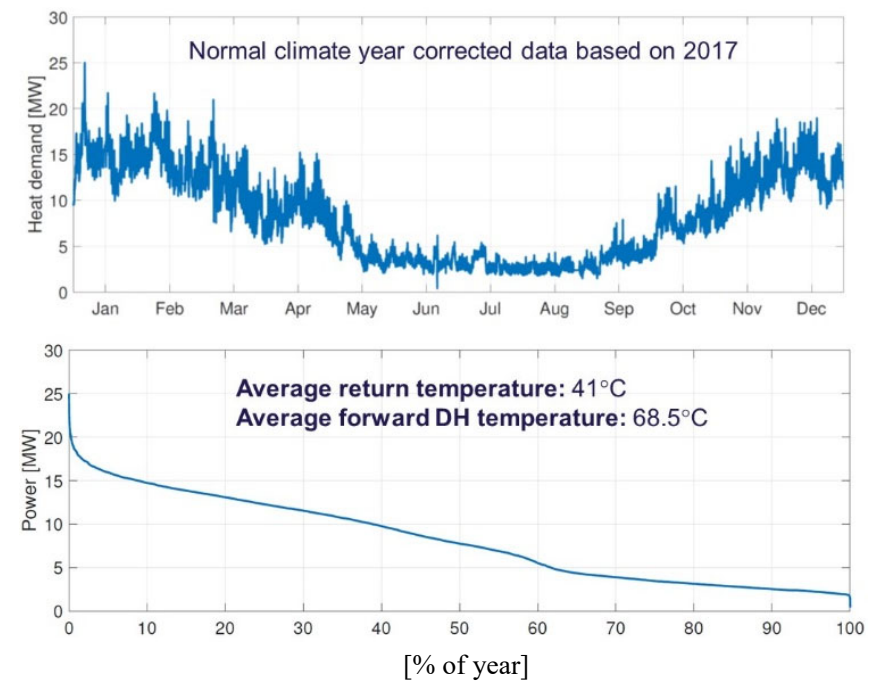

Figure 2. Corrected demand and duration curve.

The annual average forward and return temperatures of the district heating water are also shown in Figure 2. The forward temperature typically varies in the interval from $65-75{ }^{\circ} \mathrm{C}$ (in rare cases up to $80{ }^{\circ} \mathrm{C}$ ) and the return temperature varies from $36-45^{\circ} \mathrm{C}$.

Based on the local conditions in the area, it was decided that a 12-16 $\mathrm{MW}_{\text {heat }}$ maximum heat output heat pump consisting of six to eight $2 \mathrm{MW}_{\text {heat }}$ heat pumps coupled in series and a 3-5 MW electrical boiler along with a 200 MWh heat storage would be suitable to cover the load of the area. The supply system will also in the future be coupled to the centralized district-heating grid in Aarhus, which covers the peak demands.

For comparison, a conventional logical operational scheme disregarding ancillary service operation was found by establishing optimum threshold max and min limiting costs for the heat pump operation. This was established solving an MILP-problem minimizing the annual cost of system operation subject to constraining the heat demand and capacities of the individual units. The logical operational scheme is show in Figure 3.

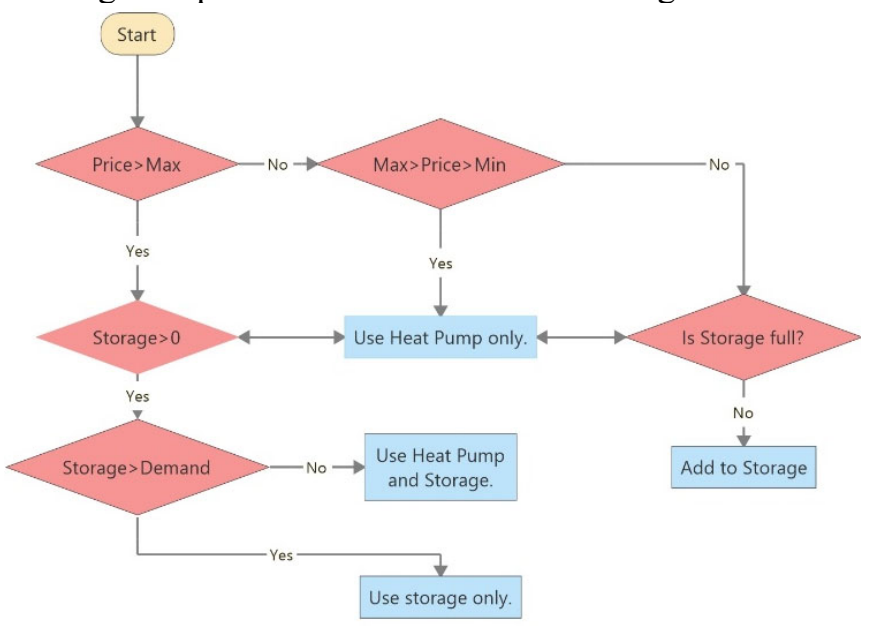

Figure 3. Logical conventional operational scheme.
Modeling a year on an hourly basis results in the following operation of the different assets shown in the following figures. Figure 4 shows the operation of the heat pumps. Each heat pump is in the modeling considered to operate by on/off control.

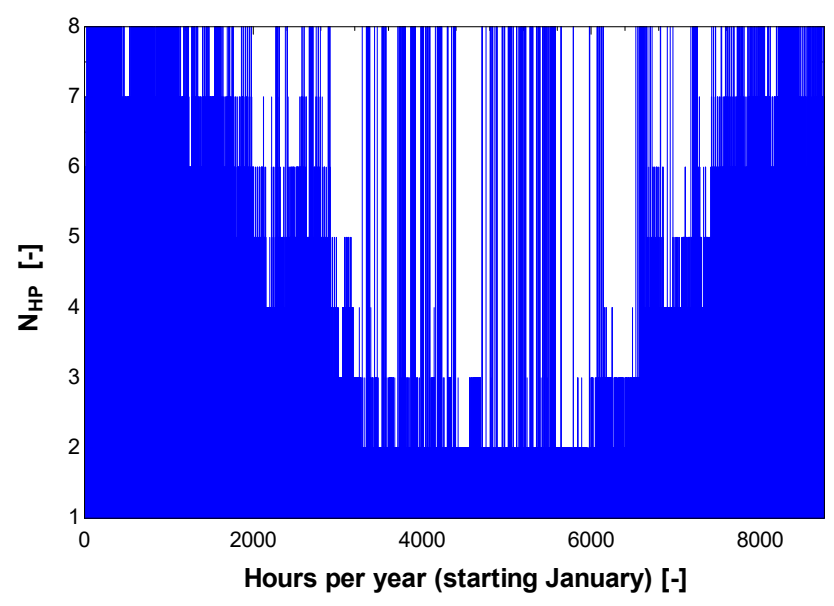

Figure 4. No. of heat pumps on/off each hour for a year.

Figure 5 shows the operation of the electrical boiler.

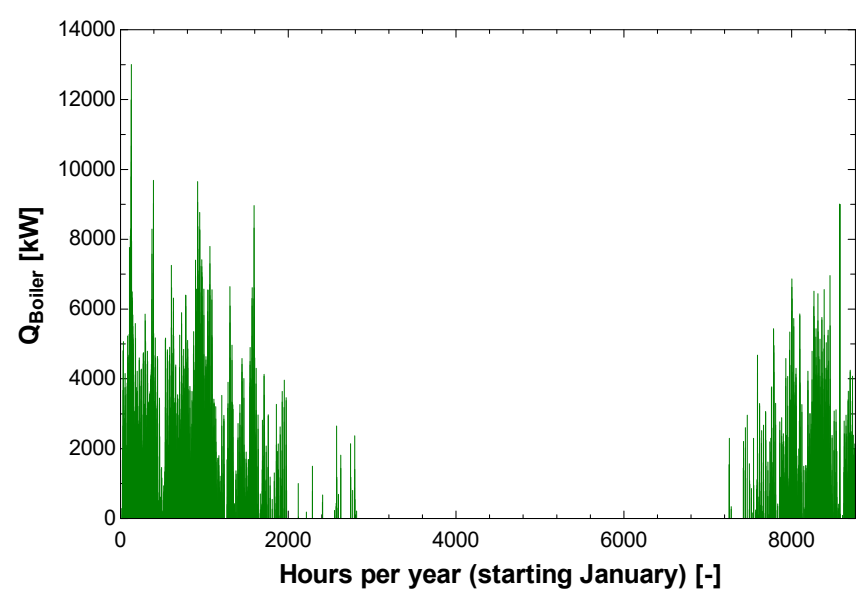

Figure 5. Operation of the electrical boiler.

It is clear the electrical boiler with 2017-prices mainly operates during the cold months in case of peak demands. The current taxation on electricity in Denmark will however in near future be changed, where the tax on using electricity for heating purposes will be lowered from $155 \mathrm{kr}$./MWh to $4 \mathrm{kr}$./MWh. This will strongly further favor the operation of electrical heating and heat pumps in the future.

Figure 6 illustrates the operation of the storage. A 200 MWh stratified hot water storage $@ 90{ }^{\circ} \mathrm{C}$ corresponds to about $3000 \mathrm{~m}^{3}$ of water, which is not a very huge thermal storage considering the system size. The reason for this is the lack of space in the district heating area. There are only very few available locations for a large storage tank. 


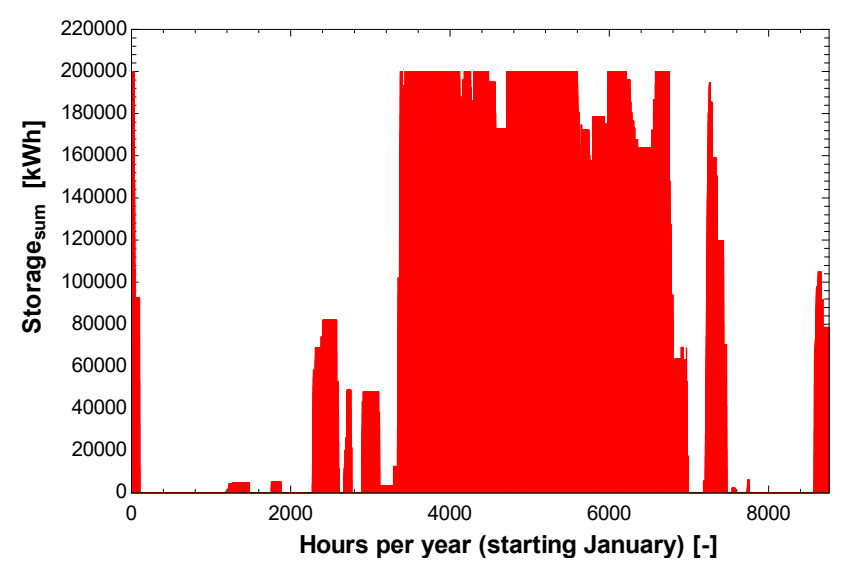

Figure 6. Operation of the storage tank.

Heat is, using the current operational scheme, typically accumulated during the Summer period. This operational pattern will clearly change significantly in case of ancillary service operations where the pattern is expected to be much more dynamic over the year. It was assumed that the storage stratification is kept at all times in this overall model. In the more dynamic case this is likely not to be true in some situations and thus a detailed model of the stratification will be implemented in the dynamic modeling. The average annual heat pump system COP was 3,52 and the overall annual cost of supplying heat for the area is 12,8 million Danish $\mathrm{kr}$.

\section{Mathematical modeling}

An ammonia-based system with flooded evaporator and two-stage screw compression was used as base case for the heat pump modeling. The process is illustrated in the PI-diagram in Figure 7 and the $\log (\mathrm{p})$,h-diagram in Figure 8:

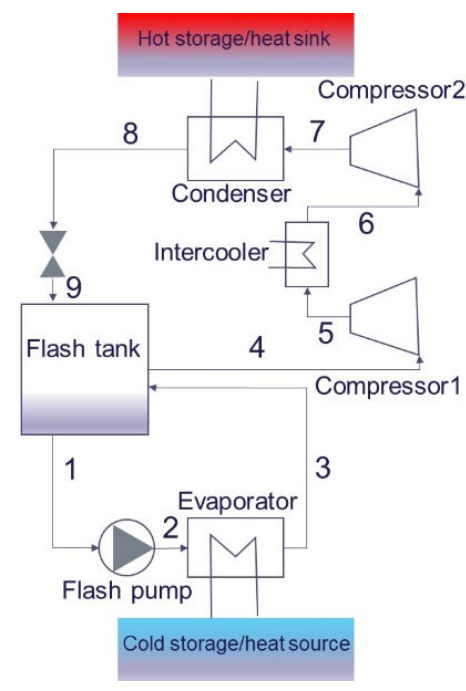

Figure 7. PI-diagram of a single heat pump.

The system can optionally be coupled with either a heat source or a "cold" thermal storage in the source-side. This storage could also receive low grade heat if other sources are available. On the sink side, normally the heat pump is coupled to a thermal storage (hot storage).

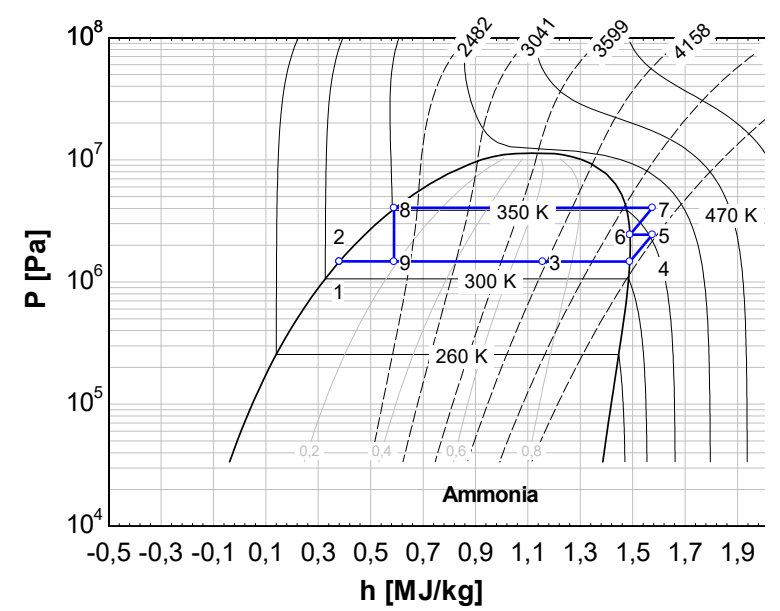

Figure 8. $\log (\mathrm{p})$, h-diagram of the heat pump process.

The steady-state models have been developed in EES (Engineering Equation Solver) which is an advanced numerical non-linear equation solver with built-in thermal property functions and the dynamic heat pump modeling was conducted using MATLAB. The steadystate models are based on the solution of non-linear equation sets coupled with thermal property models. The dynamic MATLAB model is based on the solution of a set of differential algebraic equations (DAE's). The thermal storage has been modeled one-dimensionally to account for basic stratification constraints. A discretized DAE-model was used to enable a stabile model in both filling and storage mode - see (Bitzer et al., 2008).

\subsection{Thermal/calorimetric property routine}

A simple routine using the Peng-Robinson cubic equation of state to determine thermal and calorimetric properties for the model was developed to have a fast property library for real-time emulation. The model must clearly be able to simulate faster than real time. The overall solution principle is shown in Figure 9.

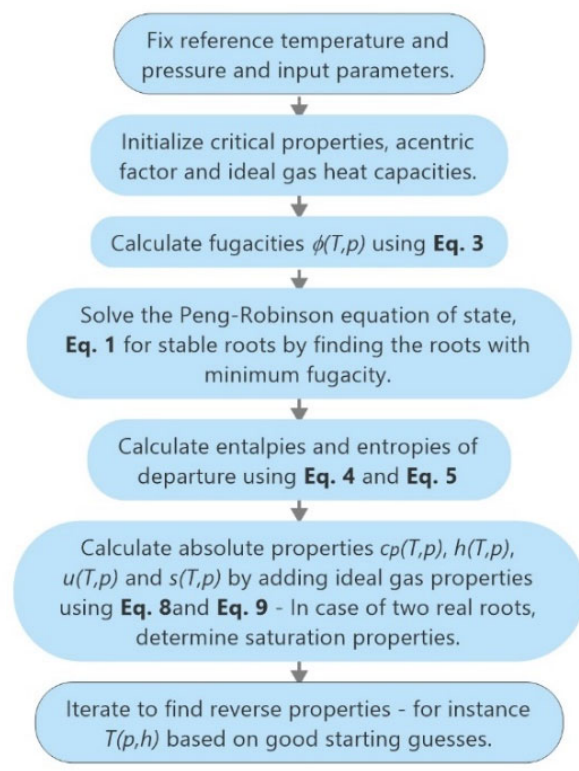

Figure 9. Use of the Peng-Robinson cubic equation of state to estimate thermal and calorimetric properties. 
Due to large inaccuracies in the liquid region for the polar fluids water and ammonia, simple regressions based on property data from EES were used in these cases. The methodology was compared to accurate data from EES based on the reduced Helmholtz equation of state for water and ammonia and for the relevant temperatures and pressures for heat pump operation, the maximum deviations was found to be less than $7 \%$ (worst case) and generally significantly lower (typically $2-3 \%$ ). This is considered acceptable for the fast dynamic model.

The generalized cubic equation of state in terms of the compressibility factor, $Z$ [-], to be solved for its roots can be written as (Matsoukas, 2012)

$$
Z^{3}-\left(B^{\prime}+1\right) Z^{2}+A^{\prime} Z-A^{\prime} B^{\prime}=0
$$

where the coefficients $B^{\prime}[-]$ and $A^{\prime}$ [-] defined as

$$
A^{\prime}=\frac{a P}{(R T)^{2}} \& B^{\prime}=\frac{b P}{R T} \text { w. } a=\frac{27}{64} \frac{\left(R T_{c}\right)^{2}}{P_{c}} \& b=\frac{1}{8} \frac{R T_{c}}{P_{c}}
$$

where $P[\mathrm{~Pa}]$ is the total pressure, $R\left[\mathrm{~J} /\left(\mathrm{mol}^{*} \mathrm{~K}\right)\right]$ is the universal gas constant, $T[K]$ is the temperature, $T_{c}[K]$ is the critical temperature and $P_{c}[\mathrm{~Pa}]$ is the critical pressure. The constants $a\left[\mathrm{~J}^{*} \mathrm{~m}^{3} / \mathrm{mol}^{2}\right]$ and $b\left[\mathrm{~m}^{3} / \mathrm{mol}\right]$ are the constants of the state equation. The fugacity coefficient $\phi[-]$ was found as:

$$
\phi=e^{\left(Z-1-\ln \left(Z-B^{\prime \prime}{ }^{\prime}\right)-\frac{A^{\prime}}{2 \sqrt{2} B^{\prime}} \ln \frac{Z+(1+\sqrt{2}) B^{\prime}}{Z+(1-\sqrt{2}) B^{\prime}}\right.}
$$

The molar specific departure enthalpies $h^{D}[\mathrm{~J} / \mathrm{mol}]$ and entropies $s^{D} \quad[\mathrm{~J} / \mathrm{mol}]$ were found analytically for the Peng-Robinson equation using eq. 4-7

$$
\begin{aligned}
& h^{D}=R T(Z-1)+\frac{T\left(\frac{d a}{d T}\right)-a}{2 \sqrt{2} b} \ln \frac{(1+\sqrt{2}) B^{\prime}+Z}{(1-\sqrt{2}) B^{\prime}+Z} \\
& S^{D}=R \ln \left(Z-B^{\prime}\right)+\frac{\left(\frac{d a}{d T}\right)}{2 \sqrt{2} b} \ln \frac{(1-\sqrt{2}) B^{\prime}+Z}{(1+\sqrt{2}) B^{\prime}+Z}
\end{aligned}
$$

where the derivative $\frac{d a}{d T}\left[J^{*} m^{3} /\left(m o l^{2} * K\right)\right]$ is found as:

$$
\frac{d a}{d T}=-0,45724 \frac{R^{2} T_{c}\left(1+\Omega\left(1-\sqrt{T_{r}}\right)\right) \Omega}{P_{c} \sqrt{T_{r}}}
$$

and $\Omega$ is

$$
\Omega=0,37464+1,54226 \omega-0,26992 \omega^{2}
$$

where $\omega[-]$ is the acentric factor.

Some corrections has been proposed to particularly (7) to obtain more accurate results. For instance (Strykjek and Vera, 1986). However, the found accuracy is acceptable for this study and the corrections would lower calculation speed which is not desirable in this application.

Specific enthalpies and entropies in $[\mathrm{J} / \mathrm{kg}]$ are then found using the reference values, the departure enthalpies and the ideal gas enthalpies as:

$$
\begin{aligned}
h & =\frac{h^{D}-h^{\text {reference }}+h^{\text {idealgas }}}{M_{\text {fluid }}} \\
S & =\frac{s^{D}-s^{\text {reference }}+s^{\text {idealgas }}}{M_{\text {fluid }}}
\end{aligned}
$$

$M_{\text {fluid }}[\mathrm{kg} / \mathrm{mol}]$ is the molar mass of the given fluid. The ideal gas enthalpies were found based on analytical integration of polynomials of the ideal gas specific heat capacities.

The Matlab-function fzero was used to find reverse properties using appropriate starting guesses and a minimization algorithm. Guesses were defined so that the algorithm converged in all cases.

\subsection{Steady-state model of the heat pump}

A steady-state model based on power and mass flow balances was setup for the system in EES. The following operational assumptions were used in the modeling of the two stage liquid overfeed ammonia based heat pump cycle:

- The district heating water was assumed to have a forward absolute pressure of 3 bar and a return pressure of 2 bar.

- Volumetric and isentropic efficiencies were variable and found using the SABROE COMP1 compressor data tool (Sabroe, 2020). For the $2 \mathrm{MW}$ system nominal value are approximately $\eta_{\mathrm{s}}=0,8$ and $\eta_{\mathrm{v}}=0,9$. The flash tank pump was assumed having an isentropic efficiency of $\eta_{\mathrm{p}}=0,8$.

- Condenser and evaporator minimum approach temperatures were fixed to $3 \mathrm{~K}$.

- Pressure drops in both the condenser and evaporator were assumed to be $3 \mathrm{kPa}$.

- In the cycle superheating was assumed to be 1 $\mathrm{K}$ and sub-cooling was $5 \mathrm{~K}$.

- The compressor displacement was $0,4428 \mathrm{~m}^{3} / \mathrm{s}$.

- The intermediate pressure between the compression stages was found optimizing the COP.

- The quality at which refrigerant leaves the liquid overfeed evaporator was fixed to $x=0,7$

The model was developed using EES's "MODULE" functionality making it easy to couple heat pumps in series and parallel on both the source and the sink side of the heat pumps. An example of serial coupling 2 heat pumps on the source and sink side, shown in Figure 10. 

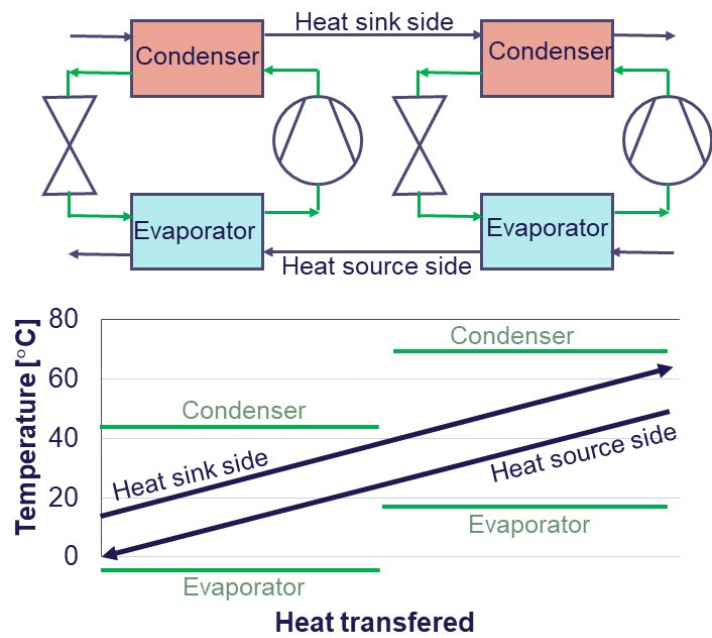

Figure 10. Serial coupled heat pump on sink/source side.

As an example, a system with 5 similar serially coupled heat pumps in terms of both condenser and evaporator having evaporation temperatures of $3 ; 7 ; 11 ; 15$ and 19 ${ }^{\circ} \mathrm{C}$ and condensing temperatures of 48;54;60; 66 and $72{ }^{\circ} \mathrm{C}$ would lead to an overall system COP of 4,46 where a single stage reference system with the same minimum and maximum temperatures would only have a COP of 3,53. The investment cost in the more complex system would of course be significantly higher, so the choice of system topology is to a large extent a choice based on the operational characteristics and possibilities. A multi-stage system would have the advantage, that the heat pumps can rapidly be decoupled which would give a rapid down-regulation response.

The heat exchangers were modeled using a basic LMTD-algoritm. A function was used to avoid numerical problems with the LMTD-formula during iterations in EES as follows (Herold et al., 2016). The returned value LMTD is the logarithmic mean temperature difference, which is a function of the four terminal temperatures $\mathrm{T} 1$ to $\mathrm{T} 4$.

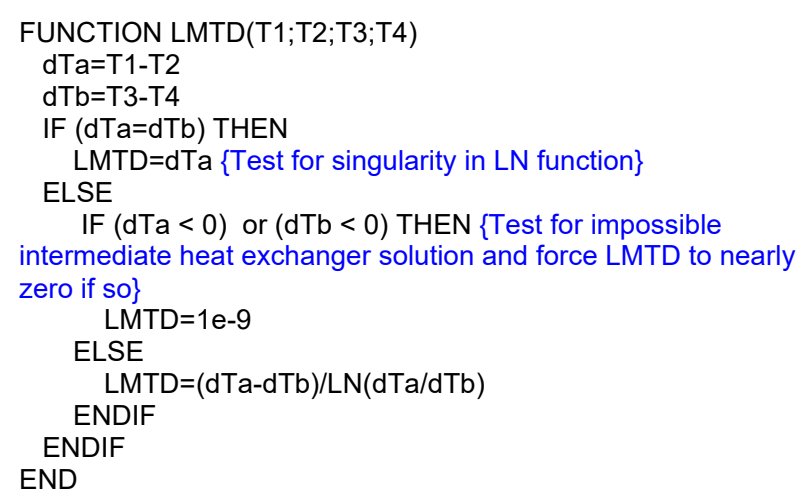

The steady-state model was used to provide inputs to the dynamic model. Thus, a number of multi-dimensional regressions were made using the generalized least squares algorithm in EES to the cycle temperatures and flows as function of the sink and source temperatures.
The regressions were compared with the rigorous model to evaluate their accuracy as shown in Figure 11.

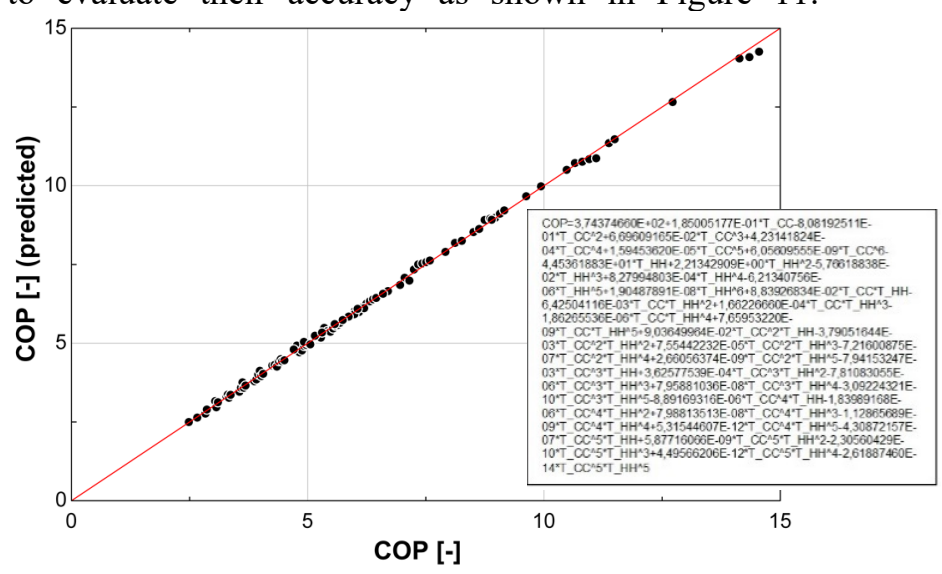

Figure 11. Example on regression comparison.

\subsection{Dynamic heat pump modeling}

The dynamic heat pump model is fundamentally based on power and mass flow balances using the dynamic forms of the first law of thermodynamics and the continuity equation. The general thermal properties are determined using the previously described steady-state model assuming relatively fast dynamics in terms of changing pressure in the system. The evaporator and condenser are considered as the primary dynamic elements, these were divided into sub-heatexchangers to model the phase change the $\varepsilon$-NTU method was used to model each of the sub-heatexchangers - see Figure 12.

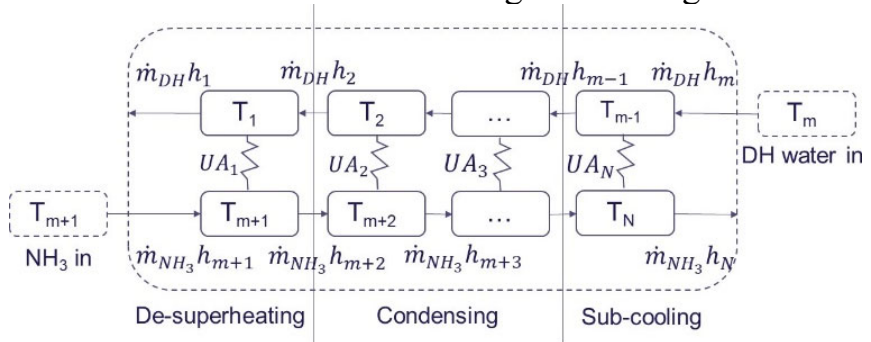

Figure 12. Discretization of latent heat exchangers.

Where $\mathrm{N} \mathrm{[-]} \mathrm{is} \mathrm{the} \mathrm{number} \mathrm{of} \mathrm{discretized} \mathrm{nodes} \mathrm{in} \mathrm{the}$ heat exchanger. A grid independency study showed that a value of $\mathrm{N}=50$ (i.e. 25 points on each side of the heat exchanger) would be reasonable for the real-time model. As illustrated, the latent heat exchangers are further divided into respectively de-superheating, evaporation and sub-cooling for the condenser and in evaporation and superheating for the evaporator.

The overall governing equations are in their discretized form in the flow direction (x) are formulated for the condenser for respectively the district heating water side $(D H)(10)$ and the refrigerant side (11)

$$
\begin{array}{r}
V_{D H} \rho_{i} \frac{d u_{i}}{d t}=\rho_{i} A_{D H} v_{i}\left(h_{i+1}-h_{i}\right)+U A_{i}\left(T_{i+m+1}-T_{i}\right) \\
V_{N H_{3}} \rho_{i+m+1} \frac{d u_{i+m+1}}{d t}=\rho_{i+m+1} A_{N H_{3}} v_{i+m+1}\left(h_{i+m}-h_{i+m+1}\right)+ \\
U A_{i}\left(T_{i}-T_{i+m+1}\right)
\end{array}
$$

Here $V\left[\mathrm{~m}^{3}\right]$ is the volume of each element, $A\left[\mathrm{~m}^{2}\right]$ is the cross sectional area at the element, $\mathrm{v}[\mathrm{m} / \mathrm{s}]$ is the fluid 
velocity, $h[\mathrm{~J} / \mathrm{kg}]$ is the specific enthalpy, $U A[W / K]$ is the total heat transmission coefficient multiplied by the element heat transfer area and $T[K]$ is the temperature. The energy balance is formulated in terms of internal energy so the equations are valid for all three heat exchanger sections. The mass balance becomes:

$$
\frac{d m}{d t}=\rho(x, t)_{i n} v(x, t)_{i n} A-\rho(x, t)_{\text {out }} v(x, t)_{\text {out }} A
$$

Energy and mass balances for the evaporator were formulated almost exactly identically as shown in (10)(12).

In each element of the discretized heat exchangers, the total heat transmission coefficient was calculated. The Dobson and Chato relation for flow condensation, as described in (Nellis and Klein, 2009) for condensation over horizontal tubes was used to estimate the convective heat transmission coefficient for the ammonia in the condensing part. For the evaporator the Shah correlations for flow boiling were used as described in (Nellis and Klein, 2009). For the remaining single-phase pipe flow regular correlations for friction factors and Nusselt numbers for developing flow in pipes considering whether the flow is laminar or turbulent was used as described in section 5.2.3. and 5.2.4 of (Nellis and Klein, 2009). It was assumed that all heat exchangers were of the shell and tube type and the heat exchanger dimensions were defined following the procedure described in (Stephan et al. 2010). The design was done considering heat exchanger pressure losses.

\subsection{Solution of governing DAE set}

The thermal relations and the sets of ordinary differential equations from the discretized heat exchangers forms a differential algebraic equation set (DAE). All algebraic equations in the DAE set are timeindependent, thus the system is of index 1 . The ODE15s solver in Matlab was used to solve the system of equations. Using this solver, all algebraic equations must be rewritten on the form of pseudo differential equations and a mass matrix $\mathbf{M}$ is defined with binary values of either 1 for differential equations and 0 for algebraic equations in the equation set. The mass matrix is practically implemented by multiplying it to the differentials as shown in (13).

$$
\boldsymbol{M}(t, y) \frac{d X}{d t}=f(t, y)
$$

The solver convergence criterions was set so the relative tolerance was $10^{-3}$ and the absolute tolerance $10^{-6}$.

\section{Results}

Results of the retrofitting of the system topology and operational optimization of the system considering steady-state operation have been investigated.
Furthermore, the dynamic capabilities and thermal and practical response time limitations in the heat exchangers of the heat pump was investigated considering both ramp-up-, ramp-down- and the response in terms of power and heat. A theoretical startup where the heating capacity is given in terms of the relative district heating water temperature is given in Figure 13 (72 ${ }^{\circ} \mathrm{C}$ DH water@100 \%):

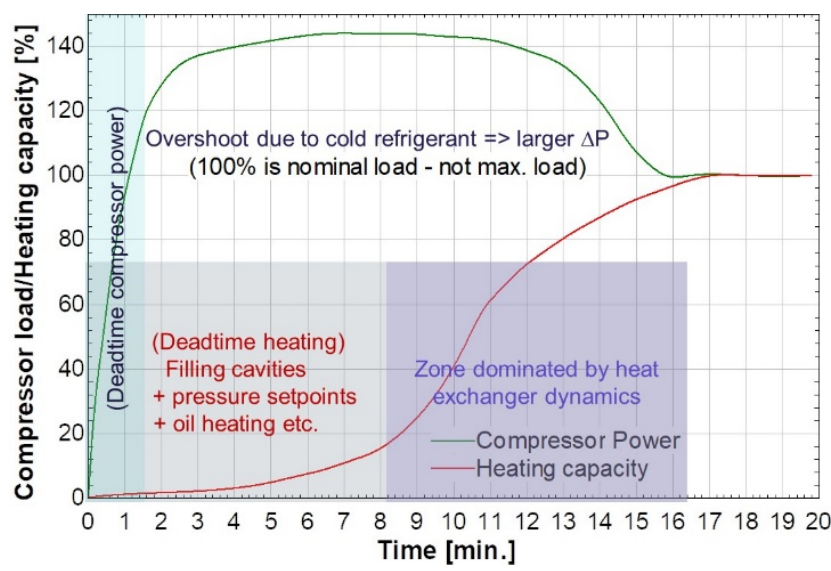

Figure 13. Typical cold-start response of the heat pump.

Nominal load refers to a heat output of 2 MW from the condenser. Max. load is the start-up capacity but at temperatures unusable for district heating. It can be seen that the start-up response in terms of heating is significantly longer than the requirements for frequency services on the power grid. Thus, operation of the heat pumps in a partial load-condition or a forecast-based scheduling would be required to meet the heat demand. In reality, the heat pumps are never operated from a cold conditions. There are typically oil separators and heat tracers installed on the compressor suction line [Johnson, 2020]. The ramping up and down of the compressor power can be significantly faster if the systems remains pre-heated or in a partial-load, so the requirements in terms of power uptake/outage can be fulfilled before steady-state is reached. The measured load-torque versus the induction motor drive torque is seen for a start-up of a screw compressor based $500 \mathrm{~kW}$ heat pump cycle in Figure 14 [Johnson, 2015].

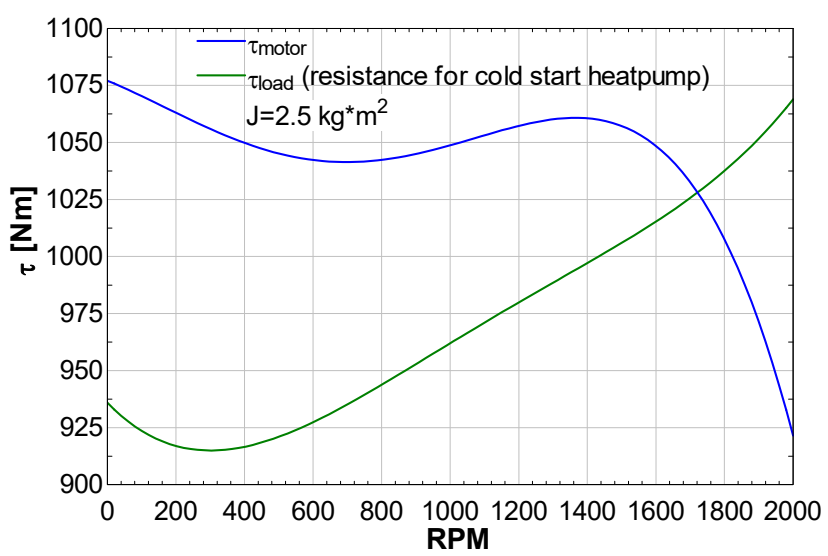

Figure 14. Load \& induction motor torque during startup. 
Solving the initial value problem shown in (14), the compressor approach to steady-state relative to the compressor RPM can be found as shown in Figure 15.

$$
J_{\text {compressor }} \frac{d \omega_{\text {compressor }}}{d t}=\tau_{\text {induction motor }}-\tau_{\text {load }, H P}
$$

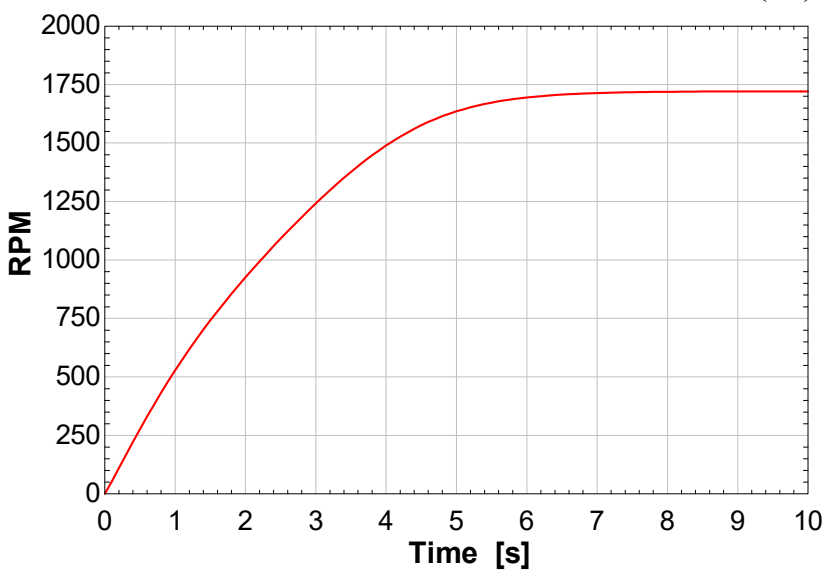

Figure 15. Approach to steady-state compressor RPM.

It can be concluded from the plot that the compressor /compressors even in worst-case does not pose a major limitation to the transient operation of a heat pump.

However, ramping down, the evaporator and condenser conditions will restrict the operation. Typical temperature curves in the condenser at steady-state are shown in Figure 16:

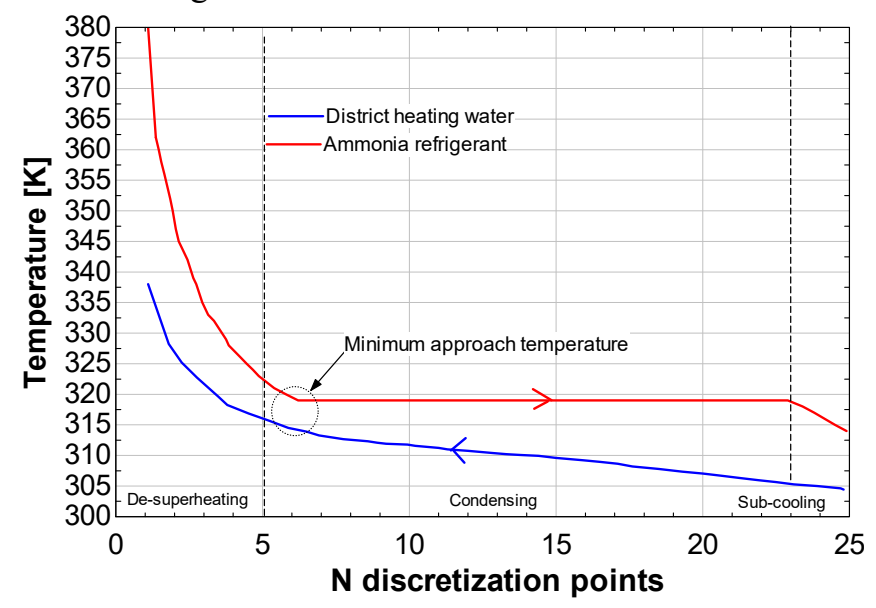

Figure 16. Condenser temperature curves.

Currently, the model has no active control scheme builtin. Thus the model is only controlled based on the initial conditions for each modeling run. Thus parameters like superheating and sub-cooling are difficult to control accurately. In future versions, control of the compressor, the pump and the valve should be implemented to address this.

The progression of the district temperature over time going from a stand-by heated condition to nearly full load (i.e. the zone dominated by the heat exchanger dynamics) is seen in Figure 17. As mentioned, the lubrication and cooling system is preheated at all times. A real cold start thus never really exist however, the heat exchangers could be cooled significantly if the system is left out of operation for longer periods during winter.

The modeling run corresponding to Figure 17 was using the following initial system conditions:

- Initial mass flow of refrigerant: $0 \mathrm{~kg} / \mathrm{s}$.

- Mass flow of liquid heat source: $90 \mathrm{~kg} / \mathrm{s}$.

- Mass flow of the district heating water: $15 \mathrm{~kg} / \mathrm{s}$.

- Initial district heating water temperature: $40{ }^{\circ} \mathrm{C}$.

- Temperature of the heat source (from a cold storage tank): $15^{\circ} \mathrm{C}$.

- Initial refrigerant temperature in the cycle: $8^{\circ} \mathrm{C}$.

- Initial refrigerant pressure in cycle: $1100 \mathrm{kPa}$.

- Initial temperature of hot source in: $15^{\circ} \mathrm{C}$.

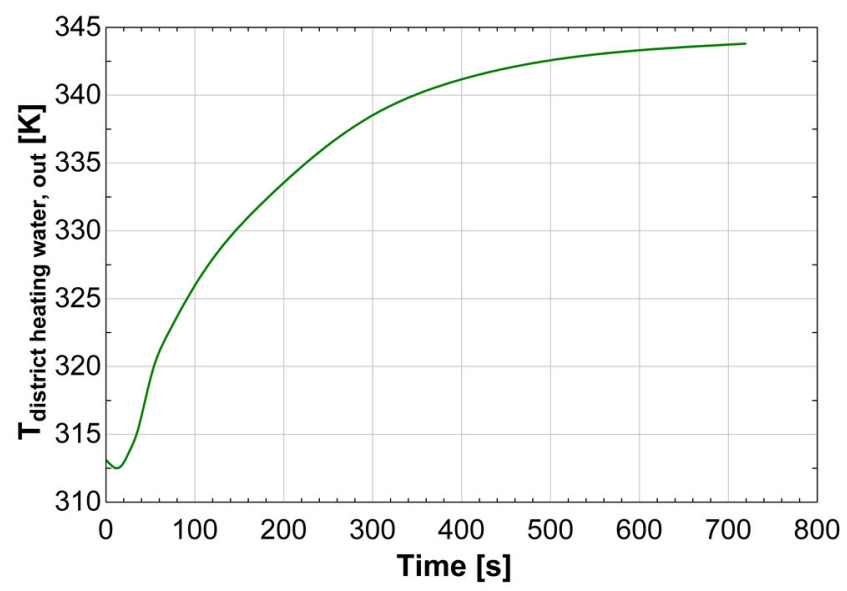

Figure 17. Dynamic response of district heating water temperature going from a "cold" condition.

It can be seen in Figure 17, that response has some deadtime in the beginning. When designing actual controllers, it is important to compensate for this. Solving the model, it was found that it in most cases solve significantly faster than real time on an ordinary Surface 6 Pro laptop computer. In rare cases, the model was slightly slower than real time but it is expected that the multicore PC's attached to the real-time emulation system at Aalborg University, Department of Energy Technology will be able to simulate significantly faster. Coupling the model with the stratified heat storage model however currently still give some challenges. The simulation times in this case increase significantly. A preliminary model simulating 48 hours of system operation including the storage has proven to be less than half as fast as real time. The models have to be optimized to address these challenges.

\subsection{Discussion of liquid overfed operation}

The liquid overfed system is often an economic tradeoff between a better heat transfer per unit volume evaporator and the cost of additional pump, piping and the flash tank. When operating the heat pump for ancillary services, there are however clear advantages of 
the liquid overfed system. When ramping down, it is important to avoid condensing of the refrigerant in the evaporator in case the system is not operated as liquid overfed. This will limit the time required to go to a partial load or stand-by situation of the heat pump. Introducing a simple energy balance for the pipematerial as shown in (15), we can assume that the saturation temperature will change rapidly with the thermal conditions of the refrigerant during ramping of the mass flow from $100 \%$ to $50 \%$ load. The temperature of the refrigerant in the evaporator will change almost instantly with the mass flow.

$$
\frac{d u_{i}}{d t}=\frac{\dot{m}\left(h_{i}-h_{i+1}\right)+\dot{Q}_{\text {suction pipe }}}{\rho_{\text {pipe }} \Delta V_{\text {pipe }}}
$$

The suction pipe thickness was assumed to be $6 \mathrm{~mm}$ thick, it is assumed adiabatic and have a specific heat capacity similar to that of stainless steel since this will be a likely material working with ammonia as refrigerant. In this calculation, it is assumed that the refrigerant leaves the evaporator with a certain degree of superheating to avoid liquid in the compressor. Figure 18 shows the results on the suction pipe temperature when ramping the heat pump linearly from $100 \%$ to $50 \%$ mass flow over the required $150 \mathrm{~s}$ for Frequency Containment Processes (FCR).

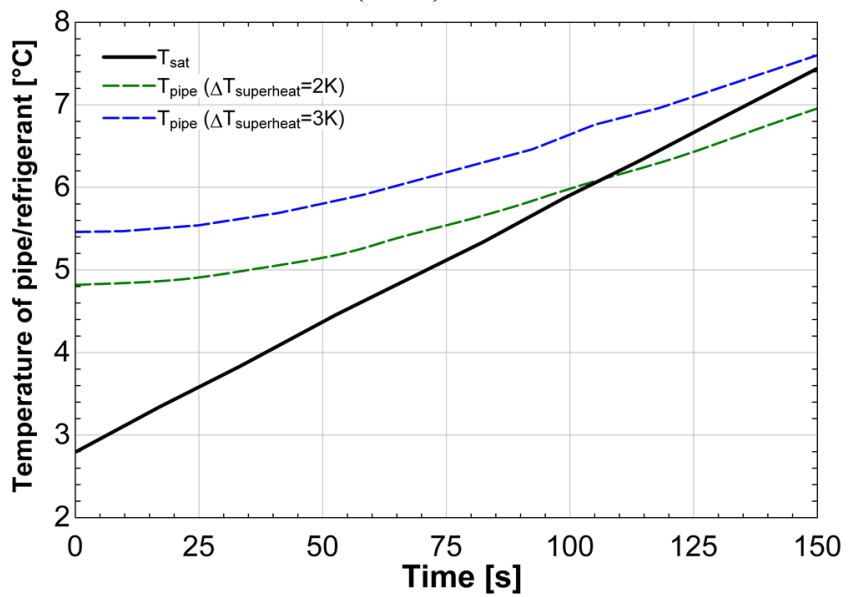

Figure 18. Ramping from $100-50 \%$ mass flow in $150 \mathrm{~s}$.

Two temperature trajectories are shown. One with a superheating of $2 \mathrm{~K}$ and one with $3 \mathrm{~K}$. These can be compared to the thick black line showing the saturation temperature of the refrigerant. The larger the superheating, the lower the cycle COP will be.

Many commercial systems are constructed with insulated suction pipes and superheating, so this could be a challenge in these cases. The problem can be resolved in multiple ways; either by increasing superheating (i.e. accepting lower COP), implementing electrical heating or constructing/ramping the system in a different manner avoiding condensation.

\section{Conclusion}

A fast numerical model of a large-scale heat pump system, which can simulate faster than real time so it can be included in the Smart Energy real-time emulation lab at Aalborg University has been developed. Different heat pump topologies have been considered in the paper and a rigorous, scalable and modularized thermal model was developed. A fundamental dynamic heat pump model has been successfully developed with focus on the detailed modeling of the latent heat exchangers. However, the model is yet to be integrated with the thermal storage model and other heat producing assets and heat distribution networks in the system and implemented in the real-time lab. Here more work still remains to have a useful modeling framework capably of solving in real time. The economic aspects of coupling heat pumps in series must also be addressed. The same goes for the considerations on a liquid overfed system. The system offer many advantages but could have a larger investment cost. Operating without a flooded evaporator however introduces challenges when ramping down the heat pump to partial load. Initial estimates indicate a undesired superheating of nearly 3 $\mathrm{K}$ in the first scompression stage suction line is required to avoid liquid formation in the evaporator in a nonliquid overfed system. More work looking into possible solutions and their consequences must be conducted.

\section{Acknowledgement}

We would like to gratefully acknowledge the Danish EUDP / ForskEL program (grant no. 12539) for funding the "Local heating for power balancing" project.

\section{References}

M. Bitzer, T. Kreuzinger, and W. Marquardt. Mathematical modelling of a domestic heating system with stratified storage tank. Mathematical and Computer Modelling of Dynamical Systems, 14 (3): 231-248, 2008.

Energidataservice.dk (homepage). Page with historical data on the cost ancillary energy services. Assessed 3/7 2020.

K. E Herold, R. Radermacher, and S. A. Klein. Absorption Chillers and Heat Pumps. CRC Press. 2016.

Johnson Controls. Engineering, Service and Maintenance Manual. SMC/TSMC and HPC 100 Mk4. 2015.

Johnson Controls. Personal communication with employees at Johnson Controls, 2020.

T. Matsoukas. Fundamentals of Chemical Engineering Thermodynamics. Prentice Hall International Series. 2013.

W. Meesenburg, W.Markussen, T. Ommen and B. Elmegaard. Optimizing control of two-stage ammonia heat pump for fast regulation of power uptake. Applied Energy. 271. 2020.

G. Nellis and S. Klein. Heat Transfer. Cambridge University Press, 2009.

Sabroe Compressor data tool COMP1. https://www.sabroe.com/en/products/sales-tools/comp1. Assessed 2/6 2020.

P. Stephan (editor). VDI Heat Atlas $2^{\text {nd }}$ edition. SpringerVerlag, Berlin, Heidelberg 2010.

R. Stryjek and J. H. Vera. PRSV: An improved PengRobinson equation of state for pure compounds and mixtures. The Canadian Journal of Chemical Engineering. 64 (2): 323-333. 1986. 